\title{
Spatial orientation from optic flow in the central visual field
}

\author{
GEORGE J. ANDERSEN and BRIAN P. DYRE \\ University of Illinois at Urbana-Champaign, Champaign, Illinois
}

\begin{abstract}
Previous research has shown that stimulation of the central visual field with radial flow patterns (produced by forward motion) can induce perceived self-motion, but has failed to demonstrate effects on postural stability of either radial flow patterns or lamellar flow patterns (produced by horizontal translation) in the central visual field. The present study examined the effects of lamellar and radial flow on postural stability when stimulation was restricted to the central visual field. Displays simulating observer motion through a volume of randomly positioned points were observed binocularly through a window that limited the field of view to $15^{\circ}$. The velocity of each display varied according to the sum of four sine functions of prime frequencies. Changes in posture were used to measure changes in perceived spatial orientation. A frequency analysis of postural sway indicated that increased sway occurred at the frequencies of motion simulated in the display for both lamellar and radial flow. These results suggest that both radial and lamellar optic flow are effective for determining spatial orientation when stimulation is limited to the central visual field.
\end{abstract}

The importance of optical information for determining spatial orientation of the observer has been investigated using a variety of measures. Some studies have used subjective measures, such as the latency and duration of induced self-motion, the angle of induced tilt of a vertical line, and magnitude estimates of the speed of induced motion. Other studies have used more objective measures, such as postural adjustment or sway.

Brandt, Dichgans, and Koenig (1973) examined the effects of the area and location of visual stimulation and angular velocity on the perception of self-motion about a vertical axis (circular vection). Subjects were required to make magnitude estimates of the speed of perceived self-motion while seated inside a large vertical drum with vertical lines painted on the inner walls. An increase in drum velocity resulted in an increase in the estimated speed of self-rotation. When stimulation was limited to the central visual field and the area of stimulation was less than $30^{\circ}$ in diameter, subjects reported little or no induced rotation. These results suggest that a wide field of view is necessary for the perception of self-motion to occur from visual stimulation.

A second study that found evidence indicating the dominance of peripheral stimulation examined induced rotation about the line of sight. Held, Dichgans, and Bauer (1975) required subjects to view monocularly sectors on

This research was supported by National Science Foundation Grant BNS 8607217. The authors would like to thank John Flach and Rik Warren for their invaluable suggestions and comments concerning the Fourier analysis. The authors would also like to thank two anonymous reviewers for comments on an earlier draft. Reprint requests should be sent to George J. Andersen, Department of Psychology, University of Illinois, 603 East Daniel Street, Champaign, IL 61820. a large rotating disk covered with a random pattern of texture elements. The retinal eccentricity measured from the point of fixation to the center of the sector varied from $18^{\circ}$ to $63^{\circ}$. Angular velocity of the disk and the area of stimulation were also varied. The subjects' task was to adjust the tilt of a line so that it always appeared vertical. Deviations of the line from true vertical were measured. The magnitude of perceived tilt increased with increasing angular velocity for velocities of $40 \% \mathrm{sec}$ and less. Perceived tilt increased with increasing retinal eccentricity, leading to the conclusion that stimulation of the periphery of the retina dominates the mechanism for orientation. Recently, Andersen and Braunstein (1985) found that stimulation of only a small area of the central visual field could result in perception of self-motion. In their study, subjects viewed displays that simulated motion of the observer along the line of sight (depth axis) through a volume of randomly positioned points. Three variables were examined: (1) the visual angle of the display $\left(7.5^{\circ}, 12.6^{\circ}\right.$, $15^{\circ}$, or $21.2^{\circ}$ ), (2) the simulated speed of observer motion, and (3) the density of texture elements in the display. The latency and duration of induced self-motion were measured. A relationship was found between the simulated speed of observer motion and the duration of perceived self-motion, with the shortest duration of selfmotion occurring for the highest speed. In addition, a significant interaction between speed and visual angle was found, with the duration of self-motion decreasing with increasing visual angle for the highest speed level. The most interesting result was that induced self-motion was reported for each visual angle condition examined. These results indicate that stimulation of an area in the central visual field of less than $10^{\circ}$ of visual angle can result in perception of self-motion. 
The studies discussed above used subjective measures, such as latency and duration of induced self-motion, magnitude judgments of the speed of induced motion, and degree of induced tilt, to investigate the perception of selfmotion. An alternative measure of spatial orientation is the adjustment of posture. If motion in the visual field is perceived as self-motion, and this percept indicates a change in the observer's speed or orientation with respect to the environment, then the observer should make postural adjustments in accordance with this change. This type of measure may be considered to be a more objective measure of spatial orientation as it does not require a subjective response by the subject.

An excellent demonstration of this effect was reported by Lee and Lishman (1975), who required subjects to stand within a large room where the walls of the room were detached from the floor and ceiling, and could be swung back and forth. In one experiment, the room was moved sinusoidally while subjects were required to stand as still as possible. Postural adjustments were measured by recording changes in the position of the observer relative to a static plate. Postural instability was greater when subjects had both eyes open than when both eyes were closed. Conditions in which the subject's normal stance was made unstable (e.g., standing on one foot) resulted in a greater effect of visual information on postural sway. These results indicate that visual information concerning spatial orientation can be used to control posture. Similar results of postural instability in young children standing in a swinging room have also been obtained (Bertenthal \& Bai, 1987; Lee \& Aronson, 1974).

Lestienne, Soechting, and Berthoz (1977) also used postural stability as a measure of spatial orientation. They measured postural stability with electromyographic recordings at the ankle. Mirrors were used to project a translating image to the peripheral regions of the visual field. The simulated motion was either a forward or a backward translation parallel to the line of sight. The magnitude of postural adjustments increased linearly with increasing velocity and density of the display, and increased with increased area of stimulation. Stimulation of the extreme periphery produced the greatest amount of body sway.

Stoffregen (1985) also examined spatial orientation using postural stability as a dependent measure. Subjects stood inside a room similar to the swinging room used by Lee and Lishman (1975). The walls were moved back and forth, while a device attached to the subject's neck measured sway. The subject's view of the moving room was masked to restrict the area of stimulation to different locations in the visual field. Subjects were required to face either the front wall, which translated parallel to the line of sight, or a side wall, which translated perpendicular to the line of sight. Using this method, either radial optic flow or lamellar optic flow exclusively could be presented to either the central or the peripheral visual field. Significant correlations between the displacement, or sway, of subjects and the movement of the walls of the room were found for peripheral stimulation (a rectangular view- ing area of $54^{\circ} \times 61^{\circ}$ was used). When lamellar flow was presented to the periphery, it produced higher correlations than when it was presented to more central regions of the visual field. Sway did not correlate significantly with the optic flow pattern for stimulation of areas less than $38^{\circ} \times 43^{\circ}$ in the central visual field. Sway also was uncorrelated with the movement of the room when radial flow was presented to the periphery. These results suggest that self-motion is dependent not only on the region of visual stimulation, but also on the type of flow information (either lamellar or radial) that is available in the visual field.

Although induced self-motion has been shown to occur from stimulation of the central visual field (within a $30^{\circ}$ diameter area around the fovea) with subjective measures, an effect of stimulation of the central visual field on spatial orientation has not been demonstrated with postural adjustment measures. There are two reasons to expect that the visual system might be sensitive to optic flow information for postural stability from stimulation restricted to the central visual field. First, there is greater sensitivity to motion in the central field (Johnson \& Leibowitz, 1974; Johnson \& Scobey, 1980). Thus, if the visual system can adjust posture on the basis of central field stimulation, it can make more finely tuned adjustments. Second, there is higher acuity in the central field than in the peripheral field. Fine spatial resolution is likely to be important for determining spatial layout of the environment and for detecting changes in the orientation of the observer with respect to the environment.

\section{EXPERIMENT 1}

Previous research (Andersen \& Braunstein, 1985) found that induced self-motion from stimulation of the central visual field could occur from radially expanding flow fields. If the perception of self-motion from stimulation limited to the central visual field is based on the expansion or contraction of flow patterns in the display, then induced self-motion may occur only for displays that include simulated translation along the depth axis. The purpose of Experiment 1 was to examine postural stability from stimulation of the central visual field when motion other than translation along the depth axis is simulated. The displays simulated a horizontal translation of the observer relative to a volume filled with randomly positioned dots.

To determine whether postural adjustments are the result of visual information present in a display, sway to that display must be compared with a baseline or control measure of sway. If a greater amount of adjustment occurs during experimental conditions than during control conditions, then one can conclude that the increased sway indicates sensitivity to visual information in the display. To make comparisons between experimental and control conditions, we employed the following methodology. Two different displays were generated, Display 1 and Display 2 . In each display the velocity of simulated observer 
motion was varied according to the sum of four different sine functions. (The reason for using the sum of four sine frequencies was to produce a pattern of velocity change that would be difficult for the subject to anticipate.) The two displays were based on two different sets of frequencies. The frequencies used for Display 1 were 0.16667 , $0.54167,0.37500$, and $0.70883 \mathrm{~Hz}$, and the frequencies used for Display 2 were $0.29167,0.45833,0.62500$, and $0.79167 \mathrm{~Hz}$. The frequency of each sine function was chosen such that it would not be a low harmonic of any other frequency used in either of the two displays.

As an example of how each display served as a control for the other display, consider one of the frequencies present in Display 1. One can compare the body sway that occurred at that frequency while the subject was observing Display 1 with the sway that occurred at that frequency while the subject was observing Display 2 , which did not contain that frequency. If the subject was responding to the visual information, sway at that frequency should be greater when the subject was observing Display 1 than when the subject was observing Display 2. On the other hand, if the tendency to sway at that frequency was unrelated to the visual display, the amount of sway at that frequency should have been no greater when the frequency was contained in the display being observed than when that frequency was not present in the display being observed. Sway at each of the four frequencies in Display 1 while observing Display 1 can thus be compared with sway at the same four frequencies while observing Display 2. Likewise, sway at the four frequencies in Display 2 while observing Display 2 can be compared with sway at those four frequencies while observing Display 1. Thus, for each of the eight frequencies, one of the displays-the one containing that frequencyserved as an experimental display and the other-the one not containing that frequency-served as a control display.

One could argue that if the frequencies examined are in the range of normal postural sway, then it is unclear whether adjustments have occurred as a result of the experimental conditions or whether the adjustments are the result of normal body sway. However, postural adjustments to a specific frequency that is not present in the driving stimulus represents an ideal baseline measure of sway. If subjects have greater sway when that frequency is present in the display than when it is not present in the display, even though the frequency is within the range of normal body sway, then it would still indicate that subjects must be adjusting their posture in accordance with the stimulus information within the display.

\section{Method}

Subjects. The subjects were 12 introductory psychology students who received course credit for their participation. All of the subjects were naive as to the purpose of the study and had normal or corrected-to-normal vision.

Design. There were two independent variables: the velocity frequencies $(0.16667,0.37500,0.54167,0.70883,0.29167,0.45833$, 0.62500 , and $0.79167 \mathrm{~Hz}$ ) and the presence or absence of a given frequency in the display (i.e., an experimental or control frequency for that display). The independent variables were run within subjects, with the display order counterbalanced across subjects.

Stimuli. The displays were simulations of motion of the observer relative to a volume of randomly positioned points. The average number of points visible in a display was 500 . The dimensions of the volume were $21 \times 21 \times 63$ units, with the projection plane 3 units from the simulated viewpoint of the observer. Only the points projected onto a 1-unit-radius circle at the center of the projection plane were plotted. One unit subtended a visual angle of $7.5^{\circ}$ for the projection size and viewing distance used. The display duration was $48 \mathrm{sec}$. The interstimulus interval was $5 \mathrm{sec}$.

To simulate observer motion, the dots were moved along a horizontal axis while the projection plane and the observer's viewpoint remained fixed. Whenever a dot moved outside the field of view, it was replaced at the opposite end along the horizontal axis at the same vertical and depth coordinates. Each display contained a velocity change that was based on the sum of four sine functions. For Display 1 the frequencies of the sine functions were 0.16667 , $0.54167,0.37500$, and $0.70883 \mathrm{~Hz}$, whereas for Display 2 the frequencies were $0.29167,0.45833,0.62500$, and $0.79167 \mathrm{~Hz}$. The amplitude of the velocity function was 18.9 units/sec for all frequencies. To minimize spurious frequency effects in the frequency analysis, the display frequencies were chosen such that two criteria were met: (1) each individual frequency must complete a whole number of cycles in the 48-sec display duration, and (2) each frequency must not be a low harmonic of any other frequency examined.

Apparatus. The displays were generated on a PDP 11-73 and were shown to the subjects on a Hewlett Packard 1310B display scope, with a resolution of $4,096 \times 4,096$. The displays were refreshed at a rate of $22.5 \mathrm{~Hz}$. A red light-emitting diode (LED) located in the center of the display scope was used as a fixation point. The eye-to-display scope distance was $.91 \mathrm{~m}$.

The subjects viewed the displays through a neutral-density glass window (11\% transmission) while standing in a large booth that surrounded the subject. A circular aperture positioned in the center of a sheet of black posterboard affixed to the window was used to restrict the field of view to $15^{\circ}$. A white annulus surrounded the aperture and was dimly illuminated from above.

Postural stability was measured by a force platform (Kistler Model 9281B). This platform measured absolute force exerted by subjects in two orthogonal directions: front to back (force along the $x$-axis) and up and down (force along the $z$-axis). The platform amplifier (Kistler Model 9861A) was adjusted to the 1-KN setting in order to record small adjustments in posture. (A force of $1 \mathrm{KN}$ is the force required to accelerate an inertial mass of $1,000 \mathrm{~kg}$ at a rate of $1 \mathrm{~m} / \mathrm{sec}^{2}$.) The sampling rate of the force platform was $12 \mathrm{~Hz}$.

Procedure. The subjects were run individually and viewed 10 trials of each display. They were instructed to stand as still as possible and fixate the red LED located at the center of the display.

\section{Results and Discussion}

The time domain data representing sway along the horizontal axis was transformed into the frequency domain using a Fast Fourier Transform (FFT). At each of the eight frequencies of interest, the amplitude of sway to the display that simulated acceleration at that frequency (experimental display) was compared with the amplitude of sway to the display that did not simulate acceleration at that frequency (control display). The amplitudes of sway for both the experimental and control conditions were converted to $z$ scores, and a two-way analysis of variance (ANOVA) was conducted using frequency and experimental/control display as the independent variables. To protect against positive biases in the $F$ tests, we computed 
the significance levels with conservative degrees of freedom (Greenhouse \& Geisser, 1959). Note that for each analysis, the actual (rather than conservative) degrees of freedom are shown. However, the $p$ values given are those for the conservative degrees of freedom (i.e., $1 / n-1$ ).

The mean postural adjustment for both the experimental and control frequencies are shown in Figure 1. A significant main effect $[F(1,11)=6.15, p<.05]$ was found between the mean sway amplitude of the eight frequencies of interest in the experimental display $(0.927 \mathrm{~mm})$ and the mean sway amplitude for the same frequencies in the control display $(0.764 \mathrm{~mm})$. This indicates that sway was induced at the specific frequencies of motion present in the displays. In addition, a significant main effect $[F(7,77)=68.62, p<.001]$ of frequency was found. According to this effect, greater amplitudes were found for the low frequencies than for the high frequencies (see Figure 1). The interaction was not significant $[F(7,77)=.3, p>.10]$.

Overall, the amplitude of sway was greater when a frequency was present in the display being observed (the experimental display for that frequency) than when that frequency was absent from the display being observed (the contro. display for that frequency). The sway amplitudes derived from the Fourier analysis, shown in Figure 1, may appear small, but it should be noted that these are based on a partitioning of the total sway into frequency components. One measure of the total magnitude of sway is the standard deviation of the subject's position of center of gravity. The average sway across subjects and conditions in the present study, using this measure, was $4.6 \mathrm{~cm}$. This value for total sway is well within the range of postural adjustment found in other studies (Lestienne et al., 1977; Winter, 1979).

The results indicate that postural adjustments occurred from stimulation of the central visual field with a lamel-

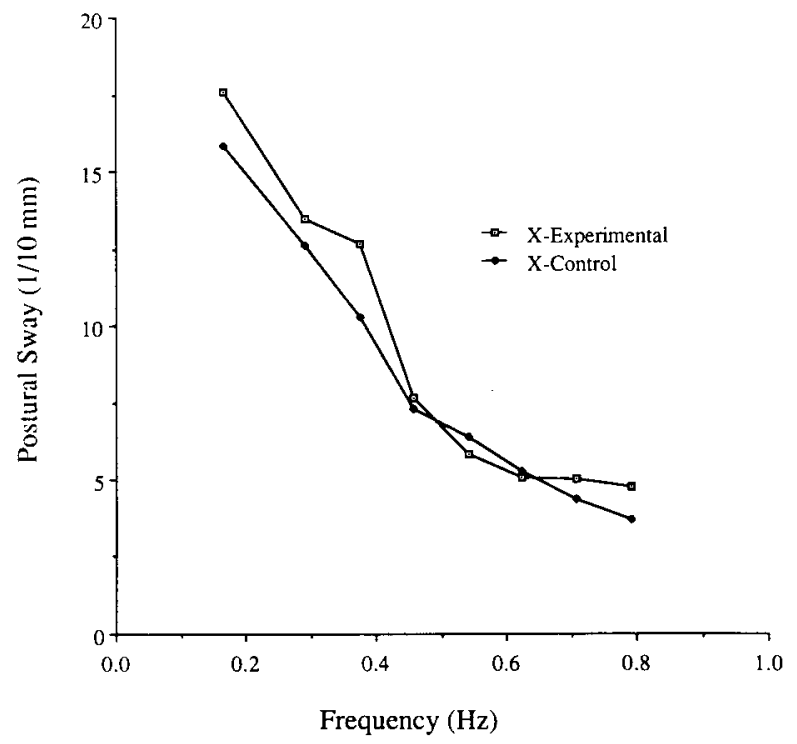

Figure 1. Effect of the frequency of the velocity function on the amplitude of postural adjustment for simulated horizontal translation. lar flow field. Stoffregen (1985) did not find postural sway to a lamellar flow field when it was presented to an area less than $38^{\circ} \times 43^{\circ}$ of the central visual field. This discrepancy in results can be attributed to several differences between the methods used in the present experiment and the methods used by Stoffregen (1985). Postural adjustments in the present study were sampled at $12 \mathrm{~Hz}$, whereas postural adjustments in the study by Stoffregen were sampled at $.5 \mathrm{~Hz}$. This suggests that the failure to find postural adjustment in Stoffregen's study may have been the result of a lower sampling rate. In addition, the measurement of postural adjustment used in the present experiment was much more sensitive than the measurement used by Stoffregen. In his study, adjustments were measured with a potentiometer attached to the subject's neck by a cord. The resolution of measurement was 10$20 \mathrm{~mm}$. The maximum amplitude of sway induced in Experiment 1 of the present study was $0.4 \mathrm{~mm}$. Thus, the failure to find adjustments from stimulation of a small field of view may have occurred because the method of measurement was not sensitive to the low amplitude of sway produced by stimulation of the central visual field.

Another difference between Stoffregen's (1985) experiments and the present experiments is the method of data analysis used. In the present experiment, a frequency analysis of postural adjustment was used to separate the amount of sway at specific frequencies from the amount of sway for all other frequencies. In addition, a comparison was made between the amount of sway for each frequency that was present in the display and the amount of sway for the same frequency when it was not present in the display. In the experiments by Stoffregen, a correlation between the pattern of motion and postural adjustment was used to analyze postural sway. This type of analysis does not allow a partitioning of sway based on specific frequencies.

Finally, another difference between the present experiment and the study by Stoffregen (1985) concerns the availability of other visual information that might have affected postural stability. In the study by Stoffregen, the visual field of the subject was occluded by a static partition. Since the partition was visible, it may have provided conflicting information that would indicate that the subject was not moving relative to the surrounding booth. In the present study, there was no conflicting visual information as the subjects viewed the displays in a dark room. This suggests that the results obtained in the study by Stoffregen when stimulation was restricted to the central visual field may have been attenuated by the presence of conflicting information.

\section{EXPERIMENT 2}

The results of Experiment 1 indicated that postural adjustment occurred with stimulation of the central visual field by lamellar flow patterns. In Experiment 2, we examined the effects on postural stability of radial flow patterns. This type of flow pattern is produced by translation along the line of sight and has been shown to induce a 


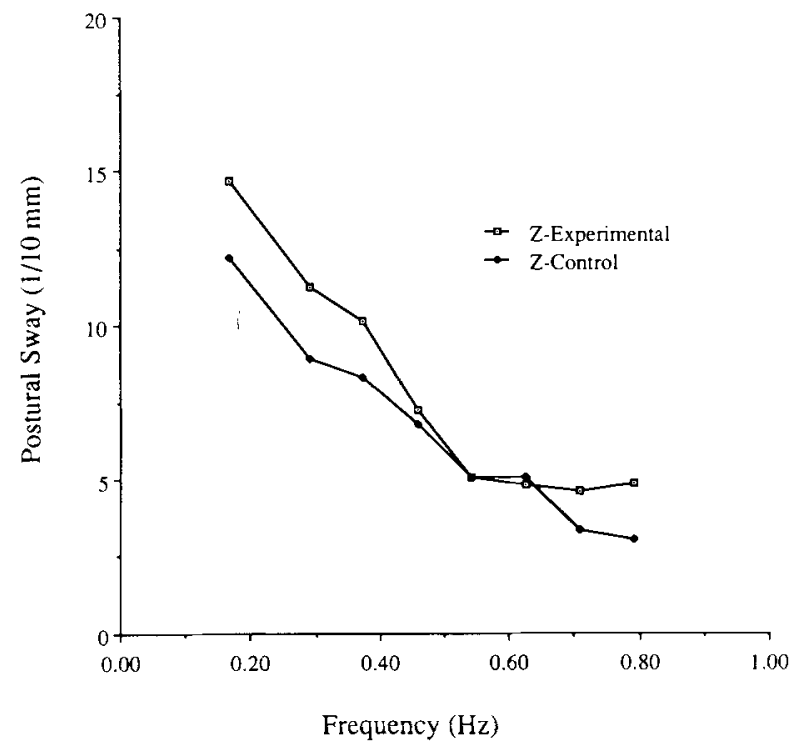

Figure 2. Effect of the frequency of the velocity function on the amplitude of postural adjustment for simulated translation along the line of sight.

perception of self-motion for small areas of stimulation of the central visual field (Andersen \& Braunstein, 1985). An examination of this type of translation will allow a comparison of postural sway effects with the results obtained with subjective measures for induced self-motion.

\section{Method}

Subjects. The subjects were 2 undergraduate introductory psychology students who received course credit for their participation and were naive as to the purpose of the study and 2 volunteers who had experience with these types of displays. All of the subjects had normal or corrected-to-normal vision.

Design. The design used in Experiment 2 was the same as that used in Experiment 1.

Stimuli. The displays used in Experiment 2 were the same as those used in Experiment 1, with the following exception: to simulate observer motion, the dots were moved along the line of sight (forward and backward) while the image plane and the observer's viewpoint remained fixed. The simulated peak velocity of the displays was 28.35 units/sec.

Apparatus and Procedure. The same apparatus and procedure were used as in Experiment 1.

\section{Results and Discussion}

The time domain data representing sway parallel to the line of sight was transformed into the frequency domain using an FFT. The amplitudes of sway for both the experimental and control conditions were converted to $z$ scores, and a two-way ANOVA was run using frequency and whether a given frequency served as an experimental or control frequency as the independent variables.

The results of Experiment 2 are shown in Figure 2. A significant main effect $[F(1,3)=18.85, p<.025]$ was found between the mean sway amplitude of the eight frequencies of interest in the experimental display $(0.774 \mathrm{~mm})$ and the mean sway amplitude for the same frequencies in the control display $(0.648 \mathrm{~mm})$. This indicates that postural adjustments occurred at the specific frequencies present in the displays.

In addition, a significant main effect $[F(7,21)=14.36$, $p<.05]$ of frequency was found, with greater amplitudes found at the low frequencies than at the high frequencies (see Figure 2). This effect is most likely due to noise created by the frequency distribution of random body sway that is present in both the experimental and control displays. No significant interaction was found between the two independent variables $[F(7,21)=0.81, p>.25]$. The total magnitude of sway (see Experiment 1) across subjects and conditions was $3.2 \mathrm{~cm}$.

\section{GENERAL DISCUSSION}

The results of Experiments 1 and 2 indicate that postural adjustments occur when stimulation is restricted to a small area of the central visual field. These results indicate that optical information from the central visual field can be used to determine spatial orientation and suggest that peripheral stimulation is not necessary for optically induced changes in perceived spatial orientation. (For a discussion of the implications of this result, see Andersen, 1986.) These results corroborate previous findings that indicated that induced self-motion could occur from stimulation of the central visual field (Andersen \& Braunstein, 1985; Post \& Chaderjian, 1988). A second important result of the present study was that postural sway was produced with both horizontal and depth translations. This suggests that the use of optic flow information from the central visual field for determining spatial orientation is not dependent on a specific type of transformation.

The present findings represent a conservative estimate of the effects of optic flow patterns in the central visual field on perceived spatial orientation. In the present study, subjects were required to stand on a static platform. Thus, both tactile and kinesthetic information indicated to the subjects that they were stationary. This information was inconsistent with visual information, which indicated to the subjects that they were changing their position in space and thus should adjust their posture. Thus, the effects of visual information on postural adjustment may have been reduced. Lee and his collaborators (Lee \& Lishman, 1975) found that a reduction of kinesthetic information for postural stability resulted in greater sensitivity to visual information. Future research should consider the role of tactile and kinesthetic information for determining spatial orientation, especially when that information is in conflict with visual information.

Previous research (Stoffregen, 1985) that examined the importance of central field stimulation found that stimulation restricted to small fields of view could not produce postural adjustments. However, a study by Lee and Lishman (1975) found indirect evidence supporting the importance of central field stimulation. Using a moving room arrangement, they compared a condition in which the subject viewed a distant surround with a condition in which the subject viewed a stationary object at a close distance. Subjects had less postural instability when viewing the 
close object than when viewing the distant surround. This result indicated that postural instability was attenuated when static objects were viewed in the central visual field. Results from the present study provide more direct evidence of the role of central field stimulation for determining postural stability and spatial orientation.

Although the interaction between frequency and the experimental and control conditions was not statistically significant, one interesting trend was the decreased separation between the experimental and control conditions for the midrange frequencies. This trend was present for both types of transformations. One possible explanation for this trend is that these frequencies are natural oscillating frequencies for stance of humans. Thus any effect at those frequencies may have been obscured by the random oscillations that occur during normal stance. Indeed, the range of frequencies investigated was well within the range of frequencies with which subjects normally sway when viewing a static environment (Yoneda \& Tokumasu, 1986). An important topic for future research would be to determine the conditions under which postural adjustments to optic flow occur at these midrange frequencies.

A second interesting result was the decrease in overall magnitude of sway with increasing frequency. This result is reasonable when one considers that at some point oscillations to high frequencies, resulting in high accelerations, cannot be maintained by the postural system. Thus, the decrease in sway as a function of frequency may represent the bandwidth limitations of the postural system. The use of a control theory approach to postural adjustment would allow the derivation of a differential equation that describes the gain and frequency limitations. However, in order to calculate such an equation, the range of frequencies must be equated according to amplitude. It is unclear whether subjects' magnitude of oscillation would be determined by peak velocity or acceleration information. Future research is needed to determine whether velocity or acceleration information is used to determine postural sway.

Finally, an important issue for future research would be the relationship between motion sensitivity in the central visual field and determining spatial orientation from optic flow. It was suggested in the introduction that the central visual field might be important in determining spatial orientation from optic flow because of the greater spatial and temporal sensitivity of the central field relative to the peripheral field. Thus, optic flow information present in the central visual field could be used to determine small changes in the velocity of the observer during loco- motion or small changes in the orientation of the observer relative to the environment. The presence of optic flow in the visual field can be the result of motion of objects in the environment relative to a static observer, motion of the observer relative to a static environment, or motion of both the observer and objects in the environment. It is likely that greater sensitivity to motion and finer spatial resolution in the central visual field may be important in determining which of these three conditions is occurring.

\section{REFERENCES}

Andersen, G. J. (1986). Perception of self-motion: Psychophysical and computational approaches. Psychological Bulletin, 99, 52-65.

ANDERSEN, G. J., \& Braunstein, M. L. (1985). Induced self-motion in central vision. Journal of Experimental Psychology: Human Perception \& Performance, 11, 122-132.

Bertenthal, B. I., \& BaI, D. L. (1987, November). Infant's postural compensations induced by central versus peripheral optical flow. Paper presented at the 28th annual meeting of the Psychonomic Society, Seattle.

Brandt, T., Dichgans, J., Koenig, E. (1973). Differential effects of central versus peripheral vision on egocentric and exocentric motion perception. Experimental Brain Research, 16, 476-491.

Greenhouse, S., \& GeIsSeR, S. (1959). On methods in the analysis of profile data. Psychometrika, 24, 95-112.

Held, R., Dichgans, J., \& Bauer, J. (1975). Characteristics of moving visual scenes influencing spatial orientation. Vision Research, 15 , 357-365.

Johnson, C. A., \& Leibowitz, H. W. (1974). Practice, refractive error, and feedback as factors influencing peripheral motion thresholds. Perception \& Psychophysics, 15, 276-280.

Johnson, C. A., \& ScoBey, R. P. (1980). Foveal and peripheral displacement thresholds as a function of stimulus luminance, line length, and duration of movement. Vision Research, 20, 709-715.

LEE, D., \& ARONSON, E. (1974). Visual proprioceptive control of standing in human infants. Perception \& Psychophysics, 15, 529-532.

LEE, D., \& LISHMAN, J. (1975). Visual proprioceptive control of stance. Journal of Human Movement Studies, 1, 87-95.

Lestienne, F., Soechting, J., \& Berthoz, A. (1977). Postural readjustments induced by linear motion of visual scenes. Experimental Brain Research, 28, 363-384.

Post, R. P., \& Chaderian, M. (1988). Circular vection is independent of retinal eccentricity. Investigative Ophthalmology and Visual Science, 29(Suppl.), 447.

STOFFREGEN, T. A. (1985). Flow structure versus retinal location in the optical control of stance. Joumal of Experimental Psychology: Human Perception \& Performance, 11, 554-565.

WINTER, D. A. (1979). Biomechanics of Human Movement. New York: Wiley.

YoNEDA, S., \& TOKUMASU, K. (1986). Frequency analysis of body sway in the upright posture. Acta Otolaryngologica, 102, 87-92.

(Manuscript received February 22, 1988; revision accepted for publication November 3, 1988.) 